\title{
The neural basis of narrative imagery: emotion and action
}

\author{
Dean Sabatinelli ${ }^{1, *}$, Peter J. Lang ${ }^{1}$, Margaret M. Bradley ${ }^{1}$ and Tobias Flaisch ${ }^{2}$ \\ ${ }^{1}$ NIMH Center for the Study of Emotion and Attention, University of Florida, PO Box 100165 HSC, Gainesville, \\ FL 32608, USA \\ ${ }^{2}$ Department of Psychology, University of Konstanz, Universitätstrasse 10, 78457 Konstanz, Germany
}

\begin{abstract}
It has been proposed that narrative emotional imagery activates an associative network of stimulus, semantic, and response (procedural) information. In previous research, predicted response components have been demonstrated through psychophysiological methods in peripheral nervous system. Here we investigate central nervous system concomitants of pleasant, neutral, and unpleasant narrative imagery with functional magnetic resonance imaging. Subjects were presented with brief narrative scripts over headphones, and then imagined themselves engaged in the described events. During script perception, auditory association cortex showed enhanced activation during affectively arousing (pleasant and unpleasant), relative to neutral imagery. Structures involved in language processing (left middle frontal gyrus) and spatial navigation (retrosplenium) were also active during script presentation. At the onset of narrative imagery, supplementary motor area, lateral cerebellum, and left inferior frontal gyrus were initiated, showing enhanced signal change during affectively arousing (pleasant and unpleasant), relative to neutral scripts. These data are consistent with a bioinformational model of emotion that considers response mobilization as the measurable output of narrative imagery.
\end{abstract}

Keywords: emotion; script imagery; fMRI

It has been proposed that narrative imagery is characterized by a network assembly of stimulus, response, and semantic or 'meaning' representations (Lang, 1979, 1985, 1994). Considering emotion as disposition toward action (Frijda, 1986; Lang et al., 1994), emotional imagery may be considered essentially equivalent to emotional responding, with overt motor aspects 'gated out.' From this perspective, emotional engagement during narrative imagery is defined by the extent of response mobilization measurable in central and peripheral nervous system. This conception has been corroborated by experimental paradigms in

\footnotetext{
*Corresponding author. Tel.: + 1-352-392-2439;

Fax: + 1-352-392-6047; E-mail: sabat@ufl.edu
}

which narrative emotional imagery, relative to neutral imagery, is accompanied by heightened physiological reactivity. This 'efferent leakage' can be demonstrated in cardiovascular, autonomic, and somato-motor systems (Lang, 1977; Miller et al., 1987; Cook et al., 1988; Vrana and Lang, 1990; McNeil et al., 1993; for a detailed review see Cuthbert et al., 1991). While heightened peripheral reactivity during narrative emotional imagery has been thoroughly demonstrated, central indices of response information processing have only begun to be explored.

Several neuroimaging studies of mental imagery have examined simple sensorimotor tasks (as opposed to dynamic, affect-relevant behavior). Focusing on the visual system, imagery paradigms 
commonly present a stimulus and ask subjects to imagine the stimulus after it has been removed. Visual cortical activation during imagery can be seen in categorically specific regions of inferior temporal lobe (O'Craven and Kanwisher, 2000; Mechelli et al., 2004) as well as functionally specific regions of occipital lobe (Kosslyn et al., 1995; Thompson et al., 2001), in one report with retinotopic consistency (Slotnick et al., 2005); however, the involvement of primary visual cortex in visual imagery is inconsistently reported (Goebel et al., 1998; Ishai et al., 2000; see Kosslyn and Thompson, 2003). Motor imagery studies have exploited similar, simple tasks, intermixing motor execution and motor imagery trials that report premotor and cerebellar activities during both tasks (Hanakawa et al., 2003; Nair et al., 2003; Solodkin et al., 2004). The involvement of primary motor areas in motor imagery has been variously reported as partial (Ehrsson et al., 2003) and absent (Dechent et al., 2004).

Mental imagery of diagnosis-relevant narratives has been explored in a few clinical studies in an attempt to understand the neural effects of therapy, which often employ narrative imagery as a desensitization method. These studies typically contrast symptom-relevant (therefore aversive) imagery with neutral imagery, and report increased activity in limbic and prefrontal cortex (Rauch et al., 1996; Shin et al., 1997, 2004; Bystritsky et al., 2001; Lanius et al., 2003). The methodologies used in the experiments were variants of narrative imagery paradigms originated in earlier psychophysiological research (Lang, 1977).

Here we investigate directly the neural concomitants of pleasant, neutral, and unpleasant narrative imagery using functional MRI (fMRI) to probe potential response-related activation that has been associated with emotional imagery. We adapted an established imagery paradigm to the scanner environment, presenting brief story-scripts to a nonclinical sample of young, healthy participants, and asked them to vividly imagine themselves engaged in the emotional and neutral scenes described while blood oxygen level-dependent (BOLD) signal is recorded throughout the brain.

We expect to identify language processing structures involved in the perceptual intake of narrative script contents, which may reflect a similar pattern of enhanced BOLD signal intensity during emotional, relative to neutral, imagery script processing, as is the case in the visual system during emotional, relative to neutral picture processing (Lang et al., 1998, Bradley et al., 2003; Sabatinelli et al., 2004, 2005).

As subjects vividly imagine the script narratives, we hypothesize that response-related neural circuitry will become active, and potentially show enhanced activation during emotional, relative to neutral contents. Past studies have included only aversive and neutral imagery scripts; therefore, differences identified may reflect differences in emotional intensity (which may be high in appetitive and aversive contexts) or emotional valence. The inclusion of pleasant as well as unpleasant imagery scripts will enable us to more specifically investigate the role of emotional arousal as well as emotional valence on brain activation.

\section{Method}

\section{Procedure}

Twenty-one introductory psychology students (11 male) at the University of Florida participated in the experiment for course credit. All volunteers consented to participate after reading a description of the study, approved by the local human subjects' review board. Before entering the scanner room, participants read all imagery scripts and recorded ratings of pleasantness and arousal, using the Self Assessment Manikin (Lang, 1980). The scripts consisted of 12 exemplars of pleasant scene contents, 6 of neutral scene contents, and 12 of unpleasant scene contents ${ }^{1}$ (see appendix for scripts).

Prior to entering the bore of the Siemens $3 \mathrm{~T}$ Allegra MR scanner, subjects were fitted with MRcompatible, shielded headphones, a button response paddle, and a patient-alarm squeezeball

\footnotetext{
${ }^{1}$ Two additional specific fear-relevant script contents (dental and snake fear) were excluded from the current analyses. These script contents were included in the design in order to allow for comparisons with clinical populations.
} 
that was positioned within easy reach. Padding and explicit verbal instruction were used to limit head motion. Once comfortable, subjects were moved into the bore, and an 8 min $3 \mathrm{D}$ structural volume was collected. T1-weighted structural scanning involved the collection of 160 sagittal slices with $1 \mathrm{~mm}$ isotropic voxels.

After a brief delay, the functional images were acquired. The functional prescription included fifty 2.5-mm-thick coronal slices $(0.5 \mathrm{~mm}$ gap) and covered the entire cortex in most subjects. The session included 580 images, with $3 \mathrm{~s}$ temporal resolution and $16 \mathrm{ml}$ voxel size $(3 \mathrm{~s} \mathrm{TR}, 35 \mathrm{~ms}$ TE, $160 \mathrm{~mm}$ FOV, $64 \times 64$ matrix). Subjects were asked to close their eyes, and imagery trials were presented in a continuous series (see Fig. 1). A trial began with an auditory script presentation for $12 \mathrm{~s}$. To limit the masking effect of scanner noise, a thin sound-deadening mat was attached to the inside of the bore, and the headphones were also shielded for sound. To be clearly audible over the background of scanner noise, yet not uncomfortable to the listener, auditory scripts were presented at $98 \mathrm{~dB}$. All subjects reported that the scripts were clear and decipherable.

Each auditory script was followed immediately by a tone signaling subjects to vividly imagine yourself fully engaged in the scene described. A second tone signaled the end of the $12 \mathrm{~s}$ imagery period, after which subjects listened to a series of nine numbers, one per second, and made a buttonpress when a prespecified number was presented. A delay of 3-9s was followed by the next trial, in pseudo-random order by content (not more than two trials of the same content in succession); the entire series lasted $29 \mathrm{~min}$. Script presentation order was unique for each subject.

\section{Data analyses}

The functional time series was motion-corrected, mean-intensity adjusted, linearly detrended, high pass filtered at $0.02 \mathrm{~Hz}$, and spatially smoothed with a $5 \mathrm{~mm}$ spatial filter using BrainVoyager QX (www.brainvoyager.com). All subjects' preprocessed functional activity was coregistered to their respective structural volumes, after transformation into standardized Talairach coordinate space (Talairach and Tournoux, 1988). These standardized functional maps were then analyzed using ANOVA to identify voxels whose intensity, after convolution with a standard hemodynamic response function, was associated with auditory script presentation as well as script imagery. A conservative false discovery rate (FDR) correction of $p<0.00001$ revealed clusters of activity associated with script presentation and script imagery, from which regions of interest (ROI) were sampled in individual subjects and broken down by script content.

ROIs were sampled from each subject's ANOVA map of significant $(p<0.01)$ script- and imageryassociated activity, within the area of interest, yet allowing the center of the $100 \mathrm{ml}$ ROI sample to be sensitive to the individual's specific neuroanatomy. Peak signal in each ROI (average percent BOLD signal $3-9$ s post script/imagery onset) for each subject was entered into an ANOVA including script content (erotica, pleasant arousing, neutral, contamination, and threat). This two-step analysis process enables conservative identification of active clusters in the average group activity map while remaining sensitive to individual differences in structural variation, which is typically lost after spatial standardization. ROI analyses also enable

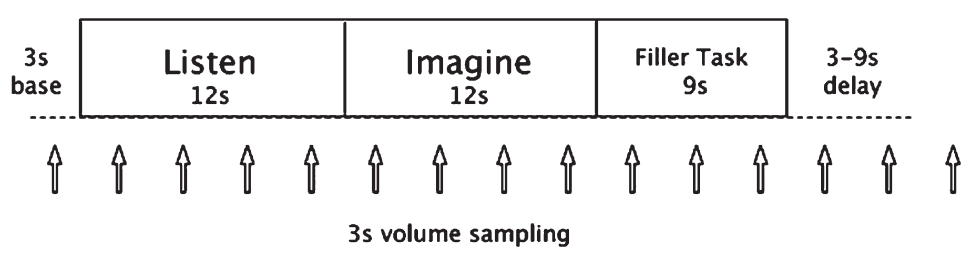

Fig. 1. Trial structure. Narrative imagery scripts were presented over headphones as subjects lay in the magnet. Immediately after script presentation, subjects imagined themselves as actively engaged in the scene described. After $12 \mathrm{~s}$, a tone signaled subjects to listen to a $9 \mathrm{~s}$ series of numbers over the headphones, and make a button-press when a predetermined number was presented. After a delay of $3-9 \mathrm{~s}$, the next trial was presented. The entire series lasted approximately $29 \mathrm{~min}$. 
Imagery Script Presentation
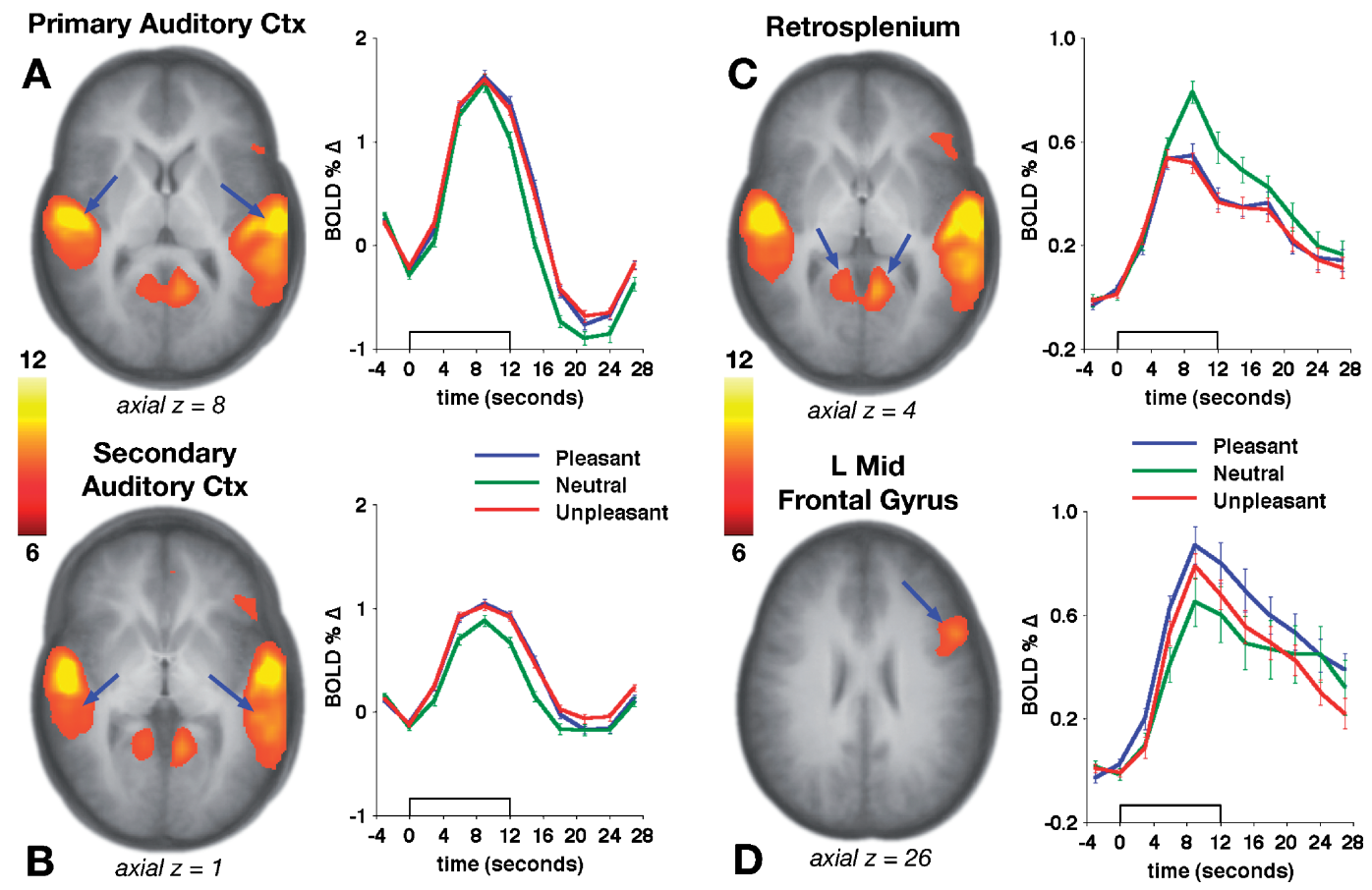

Fig. 2. Brain activation during narrative script presentation. Significant clusters of activity were evoked by auditory scripts in primary (A) and secondary auditory cortex (B) as well as retrosplenium (C) and the left MFG (D). The minimum threshold of significance in the random-effects analysis was $p<1 \times 10^{-6}$. ROI analyses from individual subjects yielded each cluster's time course of signal change, with respect to script presentation $(0-12 \mathrm{~s})$. Blue waveforms represent signal change during pleasant scripts, green during neutral scripts, and red during unpleasant scripts. Error bars represent standard errors of the mean.

the examination of event-related time courses of clusters of activity.

\section{Results}

\section{Script ratings}

The narrative scripts were rated (1-9 scale) as intended, with neutral scripts (7.0, SD 0.6) falling between pleasant (8.5, SD 0.3) and unpleasant (2.4, SD $0.6)$ scripts in terms of valence, and ranking below (3.0, SD 1.1) both pleasant (7.3, SD 1.3) and unpleasant scripts $(6.7, \mathrm{SD} 1.1)$ in terms of emotional arousal. Pleasant and unpleasant scripts were rated equivalently in emotional arousal $(t=1.20 \mathrm{~ns})$.

\section{BOLD signal during script-presentation}

The presentation of auditory scripts elicited activity in primary and secondary auditory cortex (see Fig. 2A, B, left panel). In addition, script presentation triggered activation in bilateral retrosplenial cortex (Fig. 2C, left) and left middle frontal gyrus (MFG; Fig. 2D, left). ${ }^{2}$ ROI time courses (waveform panels of Fig. 2) demonstrate that peak signal change was significantly affected by script content in secondary auditory cortex (Fig. 2B, right panel), $F(2,40)=21.75, p<0.001$, with greater signal change during pleasant and unpleasant, relative to neutral scripts (quadratic trend $F(1,20)=36.75, p<0.001)$. No effects or content interactions of hemisphere were found in tests of primary or secondary auditory BOLD signal.

\footnotetext{
${ }^{2}$ Clusters of activation during script presentation that did not meet our conservative statistical threshold were seen in ventromedial prefrontal cortex and parahippocampal gyrus. During script imagery, below-threshold clusters of activity were present in caudate, hippocampus, and posterior parietal lobe.
} 
Script content also affected signal change in left MFG, $F(2,38)=7.11, p<0.01$, again with greater signal change during pleasant and unpleasant, relative to neutral scripts (quadratic trend $F(1,19)=8.77, p<0.01)$. In retrosplenium, script valence modulated signal change as well, $F(2,34)=4.71, p<0.05$, with greater signal change during neutral relative to pleasant and unpleasant script presentations (quadratic trend $F(1,17)=7.53, p<0.05)$.

\section{BOLD signal during mental imagery}

The onset of the imagery period triggered an increase in BOLD signal in supplementary motor area (SMA; Fig. 3A), left inferior frontal gyrus (IFG; Fig. 3B), and right lateral cerebellum
(Fig. 3C). ROI waveforms (right panels of Fig. 3A-C) show that the peak level of signal change in SMA was sensitive to imagery content $F(2,38)=3.94, \quad p<0.05$, with greater BOLD signal evoked during pleasant and unpleasant, relative to neutral script imagery (quadratic trend $F(1,19)=5.02, p<0.05$ ). Left IFG showed the same sensitivity to imagery content $F(2,32)=11.91, \quad p<0.001, \quad$ with greater signal change during pleasant and unpleasant relative to neutral script imagery (quadratic trend $F(1,16)=26.50, p<0.001)$. Signal change in the right lateral cerebellum showed a marginal sensitivity to imagery content $F(2,34)=3.44$, $p=0.07$, with a trend toward greater signal change during pleasant and unpleasant, relative to neutral imagery (quadratic trend $F(1,17)=$ $3.93, p=0.06)$.

\section{Emotional Imagery}
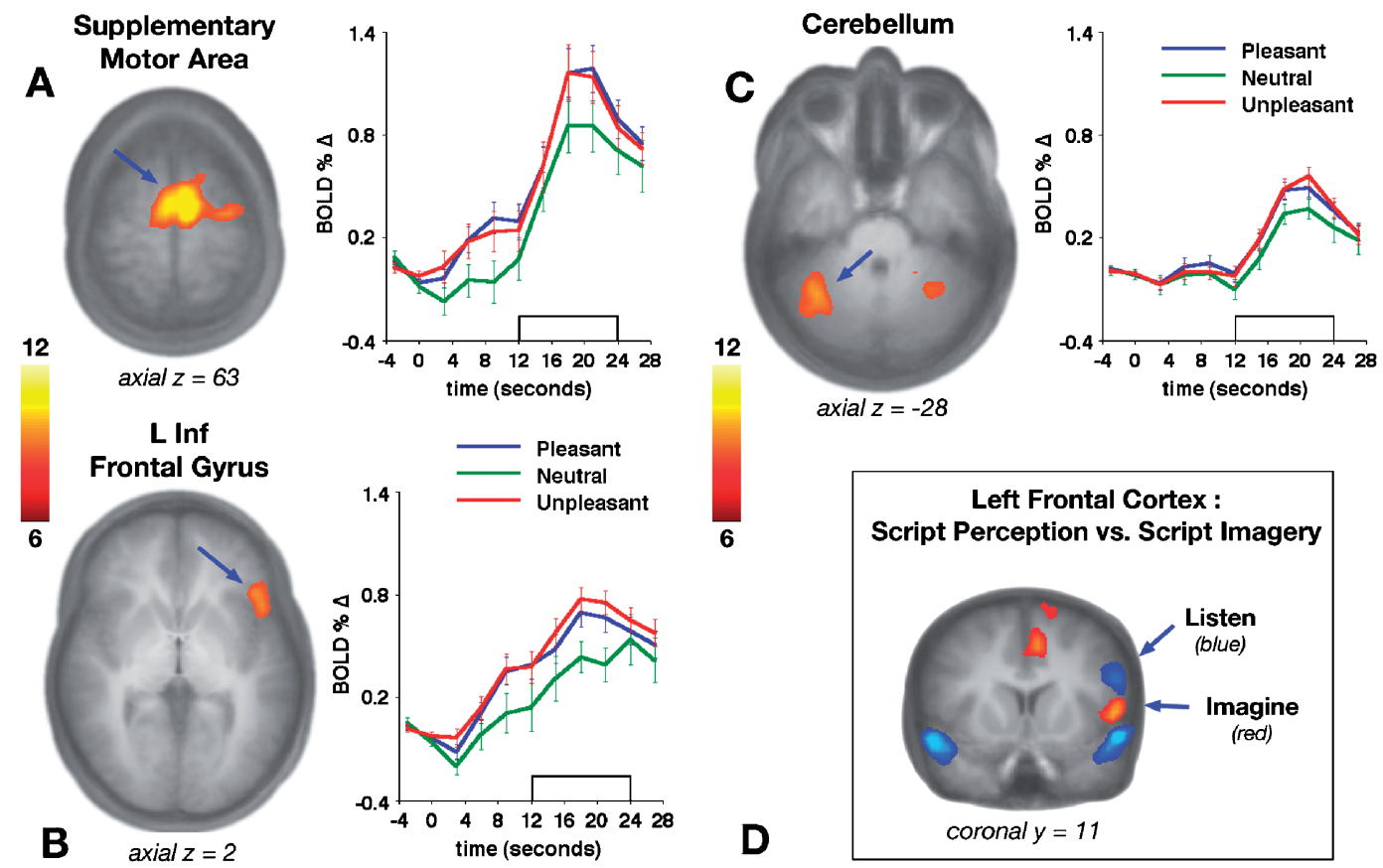

Fig. 3. Brain activity during narrative imagery. Significant clusters of activity were evoked by narrative imagery in SMA (A), left IFG (B), and right lateral cerebellum (C). The minimum threshold of significance in the random-effects analysis was $p<1 \times 10^{-6}$. Region of interest analyses from individual subjects yield each cluster's time course of signal change, with respect to the imagery period (12-24 s). Blue waveforms represent signal change during pleasant imagery, green during neutral imagery, and red during unpleasant imagery. Error bars represent standard errors of the mean. In panel D, the relative coronal locations of the left MFG cluster active during script presentation (Fig. 2D, presented here in blue), and the left IFG cluster active during script imagery (in red) are highlighted. 
Interestingly, two of the three ROIs that showed signal increases at the onset of the imagery task (SMA and IFG) also showed significant effects of script content 3-9s after the presentation of the auditory script (see Fig. 3, right panels). These signal increases were greater during pleasant and unpleasant relative to neutral script presentation (SMA, $F(2,38)=6.71, p<0.01$, quadratic trend $F(1,19)=9.36, \quad p<0.01 ; \quad$ IFG,$\quad F(2,32)=4.01$, $p<0.05$, quadratic trend $F(1,16)=4.85, p<0.05)$. The script content effect is not continuous from presentation to imagery, however, as neither SMA nor IFG shows content effects at the end of the script presentation period, prior to the onset of the imagery period (SMA, $F(2,38)=1.56 \mathrm{~ns}$; IFG, $F(2,32)=2.94 \mathrm{~ns})$.

\section{Discussion}

The act of imagining oneself engaged in a narrative recruits areas of the brain involved in planning and executing action-supplementary motor area, prefrontal cortex, and cerebellum. These effects are consistent with fMRI studies of explicit motor imagery (Hanakawa et al., 2003; Nair et al., 2003; Solodkin et al., 2004; Cunnington et al., 2005). In this dataset, it is demonstrated that the intensity of preparatory motor activation reflects in part the nature of the narrative; scenes characterized by emotional events evoke stronger signal increases. These data support a bioinformational account of emotional imagery, in which response components of associative networks are thought to be more strongly activated by emotional, than neutral narrative imagery. Prior reports of enhanced physiological mobilization observed in peripheral nervous system during emotional imagery (Lang et al., 1980; Miller et al., 1987; Cook et al., 1988; Vrana and Lang, 1990; McNeil et al., 1993) can thus be associated with response-related central nervous system correlates. Emotional imagery appears to reflect neural disposition toward action (e.g., approach, withdrawal), which can be observed as enhanced preparatory motor activation.

Immediately prior to each imagery period, subjects listened to a recorded speaker read the narrative in an emotionally muted voice. Despite the background of scanner noise, the presentation of the auditory script led to sharp and widespread BOLD signal increases in primary and secondary auditory cortex (Fig. 2). As in the visual system during picture perception (Lang et al., 1998; Bradley et al., 2003; Sabatinelli et al., 2004, 2005), the intensity of signal change in auditory association cortex was modulated by emotional intensity, i.e., the arousing pleasant and unpleasant scripts evoked (equivalently) greater signal change than did the neutral scripts. Interestingly, the primary auditory cortex did not show an effect of script emotionality at the peak of signal change, yet the descending leg of signal change (the first $6 \mathrm{~s}$ after script offset, see Fig. 2) - did show a reliable emotional arousal effect $(F(2,40)=25.02, p<0.001$, quadratic trend $F(1,20)=47.64, p<0.001)$.

Outside the auditory cortex, the activity initiated during script presentation in retrosplenium and left IFG tended to return to baseline much more slowly. This gradual decline in the BOLD signal may reflect the involvement of these structures in both the decoding and the imagination of the narrative script. It might also be a result of the tendency for subjects to begin the imagery task while the script is being presented. For the current dataset, it is defensible to suggest that the retrosplenium and IFG are involved in narrative script intake, and perhaps script imagery as well.

Beyond the timing of signal change, it is clear that the activity in retrosplenial cortex shows a unique pattern with respect to the other ROIs - a reverse arousal effect, with greater signal change associated with neutral script processing relative to pleasant and unpleasant script processing. In neuroimaging and neuropsychological studies, the retrosplenium has been closely tied to environmental navigation and orienting in large-scale space (Aquirre et al., 1998; Grön et al., 2000; for review see Maguire, 2001). We can speculate that in the absence of an emotional focus, our subjects were processing the spatial details of the imagined local environment to a greater extent. Future studies in which the degree of environmental detail is manipulated could address this issue.

The narrative scripts used in the current study were constructed to evoke pleasant, neutral, and 
unpleasant contexts, but were not explicitly controlled for the represented level of physical activity. Considering that emotion itself can be defined as disposition toward action, the separation of emotion and activity may be impossible to achieve. However, it might be possible in future work to manipulate the level of physical activity represented within each script category - to include active, inactive, and neutral as well as emotional narratives. In this way, the potentially entangled roles of action and emotion may be investigated.

The distinction between subsections of left prefrontal cortex during script presentation and script imagery (Fig. 3D) is consistent with functional distinctions commonly identified in studies of phonological and semantic language processing. In the current dataset, encoding of the narrative scripts evoked activity in a superior, posterior subsection of the left MFG that has been shown to be involved in phonological processing. During script imagery, activity increases were seen in a more inferior and anterior subsection of the left IFG, a region associated with semantic language processing (Wagner et al., 2001; Gold et al., 2005; Gitelman et al., 2005; see Hagoort, 2005). Perhaps as subjects listen to the script, content is input, but semantic elaboration is delayed until the entire text is understood and the imagery period is signaled.

Investigations of imagery-induced clinical symptoms have primarily reported activity in limbic and prefrontal structures. In panic disorder patients, relative to healthy controls, Bystritsky et al. (2001) reported limbic activation during anxiety-provoking narrative imagery, including inferior and orbitofrontal cortex, anterior and posterior cingulate, and hippocampus. Shin et al. (1997), using positron emission tomography (PET) in combat veterans with and without posttraumatic stress disorder (PTSD), identified stronger activation during trauma-related imagery in orbitofrontal cortex, insula, anterior cingulate, and amygdala. A more recent PET study (Shin et al., 2004) suggested amygdala hyperactivity in PTSD to be inversely related to hyporeactivity in medial frontal cortex. However, in a similar design, traumatized subjects with diagnosed PTSD (Lanius et al., 2003) showed relatively less anterior cingulate and thalamic activity during trauma-relevant imagery than traumatized subjects without PTSD, and no amygdala activation was reported. The variability in reports of limbic activity during traumatic imagery may in part reflect individual differences in dissociative symptoms within PTSD samples (Lanius et al., 2006). The lack of limbic activation in the current study may in part be a result of our conservative analyses, as a more liberal statistical threshold during imagery (FDR $p<0.05$ ) revealed subcortical activity in caudate and hippocampus, but no clusters in orbitofrontal cortex or amygdala. It may be that subcortical recruitment in emotional imagery is specially potentiated in clinical populations. Another possibility is that subcortical motivational circuits are more readily engaged by perceptual, rather than imagined stimuli.

In summary, these data demonstrate several effects; that auditory cortex activation reflects the emotional intensity of narrative scripts during perception. Despite the need to decipher language, affective modulation of cortical response in secondary, and perhaps primary, auditory cortex is evident while the script is heard. The current design preexposed subjects to the script stimuli prior to the experimental session, thus some of the dynamic aspects of narrative presentation were removed, potentially converting the onset of each script into a static cue for memory retrieval. Future work in which preexposure is manipulated will provide more information regarding the dependence of stimulus dynamics on this effect. In any case, this sensory cortical effect is consistent with picture processing effects in visual system, in which lateral occipital and inferior temporal visual system show reliably greater activation during affective arousing, relative to neutral picture processing (Lang et al., 1998; Bradley et al., 2003; Junghöfer et al., 2006). Thus, there may be a modality-nonspecific mechanism by which emotionally arousing stimuli evoke enhanced cortical processing.

The neural effects of narrative imagery appear to be essentially response-related. Activity in premotor, cerebellum, and prefrontal cortex show clear time-locked increases at the onset of the imagery task. Considering that response 
readiness is especially critical in emotionally charged situations, it is perhaps not surprising that emotional imagery triggered greater activation in these structures.

\begin{tabular}{|c|c|}
\hline BOLD & blood oxygen level dependent \\
\hline FDR & false discovery rate \\
\hline fMRI & functional magnetic resonance imaging \\
\hline IFG & inferior frontal gyrus \\
\hline MFG & middle frontal gyrus \\
\hline MR & magnetic resonance \\
\hline PET & positron emission tomography \\
\hline PTSD & posttraumatic stress disorder \\
\hline SMA & supplementary motor area \\
\hline
\end{tabular}

\section{Acknowledgments}

This work was supported by grants from the National Institutes of Mental Health P50 MH072850-01.

\section{Appendix}

\section{Pleasant scripts}

1. You are lying together, legs over legs, arms around bodies - kisses deep and sweet. In love on a blanket, beneath a tree, on a warm summer day.

2. You tense as the roller coaster reaches the crest. Then, you are all plunging down, screaming above the roar, together, laughing, and waving your arms.

3. As soon as you saw each other, the affair began. You remember beautiful eyes looking straight into yours - your heart in your throat, at the first touch.

4. The band is terrific. The room vibrates with sound and your skin tingles. You're dancing together, moving effortlessly with the music. You're feeling great!

5. The mountain air is clear and cold. The sun glistens on the powder as you head down the slope in gliding turns, mastering the mountain, moving with a sure, easy grace.
6. Music murmurs in the background. You're together in the big bed, naked but apart, eyes locked. You feel fingers barely touching, gliding softly along your thigh.

7. A moan of pleasure. Your body responds slowly at first, languorously, and then with a more urgent rhythm. You feel gentle hands, a soft mouth, your back arches.

8. It's a beautiful day and you're heading a new convertible to the beach. The CD player is blasting, and you're singing along at the top of your voice.

9. It's the last few minutes of the big game and it's close. The crowd explodes in a deafening roar. You jump up, cheering. Your team has come from behind to win.

10. You shiver as your bodies brush together. You reach out. You want to touch everywhere, kiss everywhere. You hear the words, 'I love you'.

11. The registered letter says 'You have just won ten million dollars!' It's amazing - You bought the winning ticket in the lottery. You cry, scream, jump with joy!

12. You are both aroused, breathless. You fall together on the couch. Kisses on your neck, face - warm hands fumbling with clothing, hearts pounding.

\section{Neutral scripts}

1. You run the comb through your hair, straighten your collar, smooth out the shirt's wrinkles. Water is running in the sink. You turn it off and leave.

2. You are relaxing on a lawn chair, looking out into the garden. A child's tricycle is abandoned on the grass. You hear the low buzz of a lawn mower in the distance.

3. It's good to be able to do nothing and just stretch out on the couch. The television is on with the sound off. You can hear the low rumble of traffic in the distance.

4. You unfold the map, spread it out on the table, and with your finger trace a route south toward the beach. You refold the map, pick up your bag, and leave. 
5. It's a quiet day without much to do. You're sitting around your place, resting, reading, and looking out the window - where leaves swirl gently in the wind.

6. You are sitting at the kitchen table with yesterday's newspaper in front of you. You push back the chair when you hear the coffee maker slow to a stop.

\section{Unpleasant scripts}

1. The garbage can is upset. Maggots crawl on the rotted food spilling out on the floor, staining the carpet. Your throat tightens with a wave of nausea, but you must clean it up.

2. A night landing in high winds: your hands clutch the seat-arms in the swaying plane. Stomach queasy. The engine coughs; stops; restarts with a strange whine.

3. A vagrant, wino, approaches you, yellow teeth and scabs on his face, clothes smelling of mold and urine. You cringe as his hand touches your sleeve.

4. As you ease the car onto the wooden bridge, it groans. In the headlights, a broken railing swings in the wind. A swift current rams against the pilings below.

5. The bathroom is filthy, toilet overflowing onto the floor spreading toward your feet. The smell is overwhelming and you run for the door.

6. You're alone in the alley in a bad part of the city. A street gang slowly surrounds you, knives out, laughing with menace. Your heart pounds as they close in.

7. It's late at night in a poorly lit parking lot. You are tense, clutching the keys. Your car stands alone in the distance, when footsteps sound behind you.

8. You are leaving the concert. (When) A drunk, smelling of smoke and alcohol, stumbles into you and throws up on your jacket. You retch, as vomit drips onto your hand.

9. You jump back, muscles tense, as the large dog strains against the chain, slobbering with teeth bared, leaping, and snarling in a crazy rage.

10. You gag, seeing a roach moving slowly over the surface of the pizza. You knock the pie on the floor. Warm cheese spatters on your shoes.

11. You bite hungrily into the hamburger, and abruptly catch the putrid smell of spoiled meat. You spit out, and a greasy piece falls down your chin onto your pants.

12. You flinch, at the screech of brakes; you look up, and see the speeding car slam into your friend. Her leg is crushed, the artery torn, and blood pumps on the road.

\section{References}

Bradley, M.M., Sabatinelli, D., Lang, P.J., Fitzsimmons, J.R., King, W. and Desai, P. (2003) Activation of the visual cortex in motivated attention. Behav. Neurosci., 117: 369-380.

Bystritsky, A., Pontillo, D., Powers, M., Sabb, F.W., Craske, M.G. and Bookheimer, S.Y. (2001) Functional MRI changes during panic anticipation and imagery exposure. Neuroreport, 12: 3953-3957.

Cook III, E.W., Melamed, B.G., Cuthbert, B.N., McNeil, D.W. and Lang, P.J. (1988) Emotional imagery and the differential diagnosis of anxiety. J. Consult. Clin. Psychol., 56: 734-740.

Cunnington, R., Windischberger, C. and Moser, E. (2005) Premovement activity of the pre-supplementary motor area and the readiness for action: studies of time-resolved eventrelated functional MRI. Hum. Mov. Sci., 24: 644-656.

Cuthbert, B.N., Vrana, S.R. and Bradley, M.M. (1991) Imagery: function and physiology. In: Ackles, P.K., Jennings, J.R. and Coles, M.G.H. (Eds.) Advances in Psychophysiology, Vol. 4. JAI, Greenwich, CT, pp. 1-42.

Dechent, P., Merboldt, K.D. and Frahm, J. (2004) Is the human primary motor cortex involved in motor imagery? Brain Res. Cogn. Brain Res., 19: 138-144.

Ehrsson, H.H., Geyer, S. and Naito, E. (2003) Imagery of voluntary movement of fingers, toes, and tongue activates corresponding body-part-specific motor representations. J. Neurophysiol., 98: 3304-3316.

Frijda, N.H. (1986) The Emotions. Cambridge, New York.

Gitelman, D.R., Nobre, A.C., Sonty, S., Parrish, T.B. and Mesulam, M.M. (2005) Language network specializations: an analysis with parallel task designs and functional magnetic resonance imaging. Neuroimage, 26: 975-985.

Goebel, R., Khorram-Sefat, D., Muckli, L., Hacker, H. and Singer, W. (1998) The constructive nature of vision: direct evidence from functional magnetic resonance imaging studies 
of apparent motion and motion imagery. Eur. J. Neurosci., 10: $1563-1673$.

Gold, B.T., Balota, D.A., Kirchhoff, B.A. and Buckner, R.L. (2005) Common and dissociable activation patterns associated with controlled semantic and phonological processing: evidence from FMRI adaptation. Cereb. Cortex, 15: 1438-1450.

Grön, G., Wunderlich, A.P., Spitzer, M., Tomczak, R. and Riepe, M.W. (2000) Brain activation during human navigation: gender-different neural networks as substrate of performance. Nat. Neurosci., 3: 404-408.

Hagoort, P. (2005) On Broca, brain, and binding: a new framework. Trends Cogn. Sci., 9: 416-423.

Hanakawa, T., Immisch, I., Toma, K., Dimyan, M.A., Van Gelderen, P. and Hallett, M. (2003) Functional properties of brain areas associated with motor execution and imagery. $\mathrm{J}$. Neurophysiol., 89: 989-1002.

Ishai, A., Ungerleider, L.G. and Haxby, J.V. (2000) Distributed neural systems for the generation of visual images. Neuron, 28: 979-990.

Junghöfer, M., Sabatinelli, D., Bradley, M.M., Schupp, H.T., Elbert, T.R. and Lang, P.J. (2006) Fleeting images: rapid affect discrimination in the visual cortex. Neuroreport, 17: 225-229.

Kosslyn, S.M. and Thompson, W.L. (2003) When is early visual cortex activated during visual mental imagery? Psychol. Bull., 129: 723-746.

Kosslyn, S.M., Thompson, W.L., Kim, I.J. and Alpert, N.M. (1995) Topographical representations of mental images in primary visual cortex. Science, 378: 496-498.

Lang, P.J. (1977) Imagery in therapy: an information processing analysis of fear. Behav. Ther., 8: 862-886.

Lang, P.J. (1979) A bio-informational theory of emotional imagery. Psychophysiology, 16: 495-512.

Lang, P.J. (1980) Behavioral treatment and bio-behavioral assessment: computer applications. In: Sidowski, J.B., Johnson, J.H. and Williams, T.A. (Eds.), Technology in Mental Health Care Delivery Systems. Ablex Publishing, Norwood, NJ, pp. 119-137.

Lang, P.J. (1985) The cognitive psychophysiology of emotion: fear and anxiety. In: Tuma, A.H. and Maser, J.D. (Eds.), Anxiety and the Anxiety Disorders. Erlbaum, Hillsdale, NJ, pp. 131-170.

Lang, P.J. (1994) The motivational organization of emotion: affect-reflex connections. In: VanGoozen, S., Van de Poll, N.E. and Sergeant, J.A. (Eds.), Emotions: Essays on Emotion Theory. Erlbaum, Hillsdale, NJ, pp. 61-93.

Lang, P.J., Bradley, M.M., Fitzsimmons, J.R., Cuthbert, B.N., Scott, J.D., Moulder, B. and Nangia, V. (1998) Emotional arousal and activation of the visual cortex: an fMRI analysis. Psychophysiology, 35: 199-210.

Lanius, R.A., Bluhm, R., Lanius, U. and Pain, C. (2006) A review of neuroimaging studies in PTSD: heterogeneity of response to symptom provocation. J. Psychiatr. Res., in press.

Lanius, R.A., Williamson, P.C., Hopper, J., Densmore, M., Boksman, K., Gupta, M.A., Neufeld, R.W., Gati, J.S. and Menon, R.S. (2003) Recall of emotional states in posttraumatic stress disorder: an fMRI investigation. Biol. Psychiatry, 53: 204-210.

Maguire, E.A. (2001) The retrosplenial contribution to human navigation: a review of lesion and neuroimaging findings. Scand. J. Psychol., 42: 225-238.

McNeil, D.W., Vrana, S.R., Melamed, B.G., Cuthbert, B.N and Lang, P.J. (1993) Emotional imagery in simple and social phobia: fear versus anxiety. J. Abnorm. Psychol., 102: 212-225.

Mechelli, A., Price, C.J., Friston, K.J. and Ishai, A. (2004) Where bottom-up meets top-down: neuronal imteractions during perception and imagery. Cereb. Cortex, 14: 1256-1265.

Miller, G.A., Levin, D.N., Kozak, M.J., Cook III, E.W., McLean Jr., A. and Lang, P.J. (1987) Individual differences in imagery and the psychophysiology of emotion. Cogn. Emotion, 1: 367-390.

Nair, D.G., Purcott, K.L., Fuchs, A., Steinberg, F. and Kelso, J.A. (2003) Cortical and cerebellar activity of the human brain during imagined and executed unimanual and bimanual action sequences: a functional MRI study. Brain Res. Cogn. Brain Res., 15: 250-260.

O'Craven, K.M. and Kanwisher, N. (2000) Mental imagery of faces and places activates corresponding stiimulus-specific brain regions. J. Cogn. Neurosci., 12: 1013-1023.

Rauch, S.L., van der Kolkm, B.A., Fislerm, R.E., Alpertm, N.M., Orrm, S.P., Savage, C.R., Fischman, A.J., Jenike, M.A. and Pitman, R.K. (1996) A symptom provocation study of posttraumatic stress disorder using positron emission tomography and script-driven imagery. Arch. Gen. Psychiatry, 53: 380-387.

Sabatinelli, D., Bradley, M.M., Fitzsimmons, J.R. and Lang, P.J. (2005) Parallel amygdala and inferotemporal activation reflect emotional intensity and fear relevance. Neuroimage, 24: $1265-1270$.

Sabatinelli, D., Flaisch, T., Bradley, M.M., Fitzsimmons, J.R. and Lang, P.J. (2004) Affective picture perception: gender differences in visual cortex? Neuroreport, 15: 1109-1112.

Shin, L.M., Kosslyn, S.M., McNally, R.J., Alpert, N.M., Thompson, W.L., Rauch, S.L., Macklin, M.L. and Pitman, R.K. (1997) Visual imagery and perception in posttraumatic stress disorder: a positron emission tomographic investigation. Arch. Gen. Psychiatry, 54: 233-241.

Shin, L.M., Orr, S.P., Carson, M.A., Rauch, S.L., Macklin, M.L., Lasko, N.B., Peters, P.M., Metzger, L.J., Dougherty, D.D., Cannistraro, P.A., Alpert, N.M., Fischman, A.J. and Pitman, R.K. (2004) Regional cerebral blood flow in the amygdala and medial prefrontal cortex during traumatic imagery in male and female Vietnam veterans with PTSD. Arch. Gen. Psychiatry, 61: 168-176.

Slotnick, S.D., Thompson, W.L. and Kosslyn, S.M. (2005) Visual mental imagery induces retinotopically organized activation of early visual areas. Cereb. Cortex, 15: 1570-1583.

Solodkin, A., Hlustik, P., Chen, E.E. and Small, S.L. (2004) Fine modulation in network activation during motor execution and motor imagery. Cereb. Cortex, 14: 1246-1255. 
Talairach, J. and Tournoux, P. (1988) Co-planar stereotaxic atlas of the human brain. 3-dimensional proportional system: an approach to cerebral imaging. Thieme Medical Publishers, Inc, New York.

Thompson, W.L., Kosslyn, S.M., Sukel, K.E. and Alpert, N.M. (2001) Mental imagery of high- and low-resolution gratings activates area 17. Neuroimage, 14: 454-464.
Vrana, S.R. and Lang, P.J. (1990) Fear imagery and the startle probe reflex. J. Abnorm. Psychol., 99: 189-197.

Wagner, A.D., Mari, L.A., Bjork, R.A. and Schacter, D.L. (2001) Prefrontal contributions to executive control: fMRI evidence for functional distinctions within lateral prefrontal cortex. Neuroimage, 6: 1337-1347. 